\title{
Effect of Intravesical Liposome-Based Nerve Growth Factor Antisense Therapy on Bladder Overactivity and Nociception in a Rat Model of Cystitis Induced by Hydrogen Peroxide
}

\author{
Tsuyoshi Majima, ${ }^{1,2}$ Pradeep Tyagi, Koji Dogishi, ${ }^{2,3}$ Mahendra Kashyap, ${ }^{2}$ Yasuhito Funahashi, \\ Momokazu Gotoh, ${ }^{1}$ Michael B. Chancellor, and Naoki Yoshimura ${ }^{2, *}$ \\ ${ }^{1}$ Department of Urology, Nagoya University Graduate School of Medicine, Nagoya, Japan; ${ }^{2}$ Department of Urology, University of Pittsburgh School of Medicine \\ Pittsburgh, Pennsylvania; ${ }^{3}$ Department of Molecular Pharmacology, Graduate School of Pharmaceutical Sciences, Kyoto University, Kyoto, Japan; ${ }^{4}$ Department \\ of Urology, Oakland University William Beaumont School of Medicine, Royal Oak, Michigan
}

The aim of this study was to evaluate whether liposome-based local suppression of nerve growth factor (NGF) in the bladder has effects on bladder hypersensitivity in a rat cystitis model induced by intravesical instillation of hydrogen peroxide (HP). HP (1.5\%) was intravesically administered to adult female Sprague-Dawley rats. Liposomes complexed with NGF antisense oligonucleotide (OND) labeled with TYE563 fluorescent tag were intravesically instilled on day 2 . Red fluorescence from the TYE 563 tag was observed with fluorescent microscopy on day 3. Four separate groups of rats were used in the following experiments: (a) sham-liposome group, (b) sham-OND group, (c) cystitis-liposome group, and (d) cystitis-OND group. Saline or $1.5 \% \mathrm{HP}$ was intravesically administered on day 0 . Empty liposomes or liposomes-antisense OND were instilled into the bladder on day 2. The following experiments were conducted to evaluate the effect of NGF antisense treatment on day 7: (a) continuous cystometry was performed in an awake condition; (b) pain behavior induced by instillation of resiniferatoxin into the bladder, including licking behavior (lower abdominal licking) and freezing behavior (motionless headturning toward lower abdomen), was observed; (c) immunohistochemical staining of the bladder and L6 DRG for NGF was performed; (d) the expression of several genes in the bladder was analyzed by reverse transcription polymerase chain reaction (RT-PCR); and (e) after Fast Blue was injected into the bladder wall, Fast Blue-positive or -negative cells in DRG neurons were separately collected by using a lasercapture microdissection method 7 days later. RT-PCR was performed to evaluate gene expressions in captured neuronal cells. The expression of TYE563 was identified only in the urothelial layer. In cystometric investigation, intercontraction intervals (ICI) were significantly $(p=0.001)$ shorter in the cystitis-liposome group in comparison to the sham-liposome group. ICI was significantly $(p=0.007)$ longer in the cystitis-OND group compared to the cystitis-liposome group. Comparisons of the shamliposome and the sham-OND groups showed no significant difference in ICI $(p=0.56)$. Licking events did not significantly differ among the four groups. In contrast, the cystitis-liposome group showed significantly more freezing events than the sham-liposome group did $(p=0.002)$. A significant reduction in the number of freezing events was observed in the cystitis-OND group compared to the cystitis-liposome group $(p=0.04)$. Immunofluorescence staining demonstrated that NGF expression in the mucosa $(p=0.02)$ and L6 DRG ( $p=0.01)$ was significantly higher in the cystitis-liposome group than it was in the sham-liposome group. The expression of NGF was significantly lower in the mucosa $(p=0.002)$ and L6 DRG ( $p=0.01)$ in the cystitis-OND group compared to the cystitis-liposome group. RT-PCR showed that the expression of $N G F$ and TRPV1 mRNA in the mucosa was significantly higher in the cystitisliposome group than it was in the sham-liposome group ( $p=0.001$ and 0.03 , respectively). On the other hand, these gene expressions were significantly lower in the cystitis-OND group than they were in the cystitis-liposome group ( $p=0.007$ and 0.02 , respectively). The cystitis-liposome group showed significantly higher expression of TRPA1, $P 2 X 3$, and $B D N F$ mRNA in labeled bladder afferent neurons than

${ }^{*}$ Correspondence: Dr. Naoki Yoshimura, Department of Urology, University of Pittsburgh School of Medicine, Suite 700, 3471, Fifth Ave, Pittsburgh, PA 15213. E-mail: nyos@pitt.edu 
the sham-liposome group did ( $p=0.03,0.01$, and 0.001 , respectively). These gene expressions were significantly lower in the cystitis-OND group compared to the cystitis-liposome group $(p=0.04,0.006$, and 0.03 , respectively). The study indicated that intravesical application of liposome-NGF antisense OND significantly improved bladder hypersensitivity induced by chemical cystitis in rats. Intravesical treatment with liposome-OND conjugates could be a novel local therapy of hypersensitive bladder disorders such as bladder pain syndrome/interstitial cystitis.

Keywords: nerve growth factor, antisense, liposome, cystitis, nociception, rat

\section{INTRODUCTION}

INTERSTITIAL CYSTITIS/BLADDER PAIN SYNDROME (IC/ BPS) is a chronic bladder disease with various degrees of inflammatory changes, with which patients present with lower urinary tract symptoms, including pelvic pain and urinary frequency. ${ }^{1,2}$ Although it substantially reduces patients' quality of life, the etiology remains unclear. However, there has been increasing evidence to support that increased excitability of bladder afferent pathways is involved in the pathophysiology of IC/BPS. ${ }^{3-6}$

Nerve growth factor (NGF) is known to be one of the neurotrophins that plays a crucial role in cell growth, differentiation, regeneration, neurotransmitter function, development, and phenotypic maintenance of neurons in the peripheral nervous system. ${ }^{7}$ NGF is also recognized as a complex regulator of afferent plasticity in response to injury or inflammation, and has been implicated in diverse pathologies such as visceral pain, rheumatoid arthritis, and bronchial asthma. ${ }^{8}$ Furthermore, according to a previous meta-analysis study, patients with IC/BPS have higher urinary NGF and NGF/creatinine levels compared to healthy people. ${ }^{9}$ Moreover, previous animal studies have shown that NGF in the bladder was upregulated in rat cystitis models induced by cyclophosphamide ${ }^{10}$ or acetic acid. ${ }^{11}$ These findings led to a randomized double-blind placebo-controlled Phase 2 study, which examined the effects of tanezumab, an anti-NGF monoclonal antibody, on clinical symptoms in IC/BPS patients, and showed a significant improvement in pain and urgency after systemic administration of the NGF monoclonal antibody in IC/ BPS patients. ${ }^{12}$ However, another clinical trial reported a severe systemic adverse effect, which led the Food and Drug Administration to terminate other trials using systemic application of NGF antibodies. ${ }^{13}$ Thus, local anti-NGF therapy would be ideal to reduce the systemic toxicity. In this regard, an intravesical liposome-based NGF antisense oligonucleotide (OND) therapy was developed to demonstrate that liposome-mediated NGF antisense delivery significantly reduced NGF expression in the bladder, resulting in the improvement of bladder pain behavior and overactivity in rat models of acetic acid-induced acute cystitis ${ }^{11}$ or experimental colitis. ${ }^{14}$ However, it remains unclear whether an intravesical liposomal-OND treatment targeting the bladder NGF expression has beneficial effects in a long-lasting cystitis condition.

It has been reported that a single intravesical instillation of hydrogen peroxide (HP) induces relatively long-lasting bladder inflammation, bladder overactivity, and nociceptive behavior in mice for up to 14 days. ${ }^{15,16} \mathrm{HP}$ is a known reactive oxygen species. According to previous studies, HP upregulates the expression of $\mathrm{p} 53,{ }^{17}$ which could activate tumor necrosis factor alpha (TNF- $\alpha)^{18}$ and inflammasomes ${ }^{19}$ to prompt tissue inflammation. In animal models of cystitis induced by HP, certain characteristics such as bladder histology, cytokine production, and increased urothelial permeability were similar to those in IC/ BPS. ${ }^{15,16}$ Hence, this study investigated whether intravesical application of liposome-NGF antisense OND conjugates had effects on bladder hypersensitivity in a rat cystitis model induced by intravesical instillation of $\mathrm{HP}$, whose inflammatory conditions are longer-lasting compared to other conventional acute cystitis models.

\section{METHODS}

All animal experiments were performed according to the experimental protocol approved by the University of Pittsburgh Institutional Animal Care and Use Committee (IACUC). Nine-week-old Sprague-Dawley female rats were purchased from Harlan Laboratories (Pittsburgh, PA).

\section{Reagents}

The 18mer phosphorothioated antisense OND was designed with the sequence 5'-GCCCGAGA CGCCTCCCGA3 $3{ }^{11,14}$ For the evaluation of bladder uptake of antisense OND, the OND with the same sequence was labeled by a $5^{\prime}$ tag of TYE 563 . Cationic liposomes were prepared as follows: 1, 2dioleoyl-3-trimethylammonium-propane (Avanti Polar Lipids, Inc., Alabaster, AL) was dissolved and mixed in chloroform to obtain a homogenous mixture of lipids. After the solvent was evaporated 
with a dry nitrogen, it was thoroughly dried up by a vacuum pump overnight so that a thin lipid film was yielded. Nuclease free water was added to the lipid film with a final lipid concentration of $7 \mathrm{mM}$. The mixture was sonicated until it became transparent. The antisense OND $(12 \mu \mathrm{M})$ was complexed with liposomes by incubation at room temperature for $30 \mathrm{~min}$.

\section{Bladder uptake}

A polyethylene catheter (PE) 10 was transurethrally inserted into the bladder to empty the bladder under isoflurane anesthesia on day 0. HP solution (1.5\%, $0.3 \mathrm{~mL}$; Sigma-Aldrich, St. Louis, MO) was then administered into the bladder through the catheter. Rats were first held in the supine position, followed by the changes to the right lateral position and then to the left lateral position for $10 \mathrm{~min}$ at each position. Thereafter, the bladder was completely emptied. Sham rats were injected with saline without HP into the bladder. For the identification of liposome-OND, fluorescent TYE 563-conjugated OND complexed with liposomes $(0.3 \mathrm{~mL})$ was intravesically administered and kept for $1 \mathrm{~h}$ under isoflurane anesthesia on day 2, and the rats were sacrificed on day 3 . The bladder was immediately harvested for cryosectioning into $10 \mu \mathrm{m}$ sections. The expression of TYE 563 was observed under a fluorescent microscope.

In another set of experiments, rats were divided into four groups: (a) sham- liposome group with an intravesical treatment of empty liposome without cystitis, (b) sham-OND group with an intravesical liposome-OND treatment without cystitis, (c) cystitis-liposome group with intravesical HP and empty liposome treatments, and (d) cystitis-OND group with intravesical HP and liposome-OND treatments. Either saline or $1.5 \% \mathrm{HP}$ solution ( $0.3 \mathrm{~mL}$ each) was administered into the bladder on day 0 for sham or cystitis rats, respectively. Empty liposomes or $12 \mu \mathrm{M}$ of antisense OND complexed with liposomes were instilled into the bladder on day 2 for liposome or liposome-OND treatment, respectively. The following experiments were then performed on day 7. In each treatment group, cystometry, nociceptive behavior measurements, histochemical analysis, and reverse transcription polymerase chain reaction (RT-PCR) were performed in separate subgroups of animals.

\section{Cystometry}

A PE 50 tube was inserted into the bladder via the dome under isoflurane anesthesia. The catheter was connected via a three-way stopcock to a pressure transducer and to a syringe pump. Physiological saline was continuously unfused into the bladder at a rate of $0.04 \mathrm{~mL} / \mathrm{min}$ after the rats recovered from anesthesia. Intravesical pressure was recorded with Chart 5 software (AD Instruments, Milford, MA). Cystometric parameters such as residual volume (RV), voiding efficiency (VE), basal pressure (BP), micturition threshold (MT), peak pressure (PP), intercontraction intervals (ICI), and bladder compliance were examined according to the methods in a previous study. ${ }^{20}$

\section{Bladder weight}

After cystometry, the bladder was harvested from each rat and weighed.

\section{Nociceptive behavior observation}

In order to evaluate bladder pain sensitivity, resiniferatoxin (RTx), a TRPV1 agonist, was instilled into the bladder, and bladder-derived nociceptive behaviors were observed. As previously reported, two types of nociceptive behavior-licking (lower abdominal licking) and freezing (motionless head-turning to the lower abdomen)could be observed after intravesical administration of RTx. It was shown that licking behavior tended to be derived from nociceptive stimulation of the urethra carried via the pudendal nerve, whereas freezing behavior was likely to be induced by pelvic nerve stimulation. ${ }^{21,22}$ The rats were acclimated in a metabolic cage for at least $2 \mathrm{~h}$ before nociceptive behavior studies. A PE 10 catheter was inserted into the bladder to drain urine. After $3 \mu \mathrm{M}$ of RTx (Sigma-Aldrich) was intravesically instilled through the catheter at a volume of $0.3 \mathrm{~mL}$ and kept for $1 \mathrm{~min}$, the rats were placed back into the metabolic cage. Thereafter, the number of licking and freezing events was counted by a blinded observer for a $15 \mathrm{~min}$ period divided into $5 \mathrm{~s}$ intervals. When licking or freezing events were observed during a $5 \mathrm{~s}$ interval, they was scored as one positive event.

\section{Hematoxylin and eosin staining}

After the rats were perfused through the left ventricle with cold heparinized-physiological saline, followed by $4 \%$ paraformaldehyde (JT baker), the bladder and L6 DRG were harvested on day 7. Tissues were post-fixed with $4 \%$ paraformaldehyde overnight and incubated with $20 \%$ sucrose for cryoprotection for $48 \mathrm{~h}$. Thereafter, they were embedded into the cryo-embedding media (OCT; Sakura Finetek USA, Inc., Torrance, CA) and frozen. The frozen bladder tissue was cut at a thickness of $10 \mu \mathrm{m}$ (transverse sections) using a cryostat and 
then stained with hematoxylin and eosin or antiNGF antibody.

\section{NGF staining}

After blocking with $20 \%$ goat serum (Vector Laboratories, Inc., Burlingame, CA) containing $0.2 \%$ Triton X-100 (Sigma-Aldrich) for $2 \mathrm{~h}$, cryosections were incubated with primary rabbit polyclonal antiNGF antibody (1:50; Santa Cruz Biotechnology, Dallas, TX) overnight at $4^{\circ} \mathrm{C}$. The sections were washed four times with phosphate-buffered saline containing $0.1 \%$ goat serum and $0.1 \%$ Triton X-100. Next, secondary goat anti-rabbit Alexa Fluor 488 antibody (1:200; Santa Cruz Biotechnology) was applied for $2 \mathrm{~h}$. The sections were mounted with aqueous mounting medium. NGF immunoreactivity was visualized, and images were captured with a fluorescence microscope. The digital image data were imported to the Image $J$ image analysis software (National Institutes of Health). In order to quantify NGF immunoreactivity in the mucosal and detrusor layers separately, a freehand drawing tool was used to select each layer to determine the total pixel area. Then, the threshold was determined as the pixel intensity above the background level of the same section and applied to the region of the interest. NGF immunoreactivity was analyzed by the percent ratio of the area above the threshold relative to the total area in each section and then averaged in the bladder sections. To quantify the number of NGF-positive cells in L6 DRG sections, a threshold was applied to the region of the interest in the same manner. A cell with a higher intensity than the threshold was defined as an NGF-positive cell. NGF immunoreactivity was analyzed by the percent ratio of the number of NGFpositive cells relative to the total number of neuronal cells in L6 DRG sections. The average number of NGF-positive cells per DRG section was calculated.

\section{Gene expression in the bladder}

Bladder tissues were harvested and separated into mucosal and detrusor layers on ice under a microscope. Each tissue was homogenated with Trizol (Thermo Fisher Scientific, Waltham, MA). Then, total RNA was extracted using an RNeasy Mini Kit (QIAGEN, Valencia, CA) according to the manufacturer's manual. Next, it was transcribed to cDNA using a High-Capacity cDNA Reverse Transcription Kit (Thermo Fisher Scientific). mRNA levels were quantified with an Mx3000P ${ }^{\mathrm{TM}}$ Real-Time PCR System using SYBR ${ }^{\circledR}$ Green PCR Master Mix. cDNA was amplified for 40 cycles $\left(95^{\circ} \mathrm{C}\right.$ for $15 \mathrm{~min}, 94^{\circ} \mathrm{C}$ for $30 \mathrm{~s}$, and $55^{\circ} \mathrm{C}$ for $60 \mathrm{~s}$ ). The sequences of used primers are shown in Supplementary Table S1 (Supplementary
Data are available online at www.liebertpub.com/ hum). The specificity of each primer was confirmed by a melting curve analysis. Each mRNA expression, with fold differences determined by the $\Delta \Delta \mathrm{CT}$ method, was compared among the four groups.

\section{Gene expression in L6 DRG}

In order to evaluate mRNA expression in bladder afferent neurons obtained from L6 DRG, 2\% w/ $\mathrm{v}$ Fast blue (Polysciences, Inc., Warrington, United Kingdom) was injected into four sites of the bladder wall ( $5 \mu \mathrm{L} /$ site, total $20 \mu \mathrm{L})$ under isoflurane anesthesia on day 0 using another set of four groups of animals. After L6 DRG was harvested on day 7, they were embedded into the OCT compound and frozen. The frozen tissue was cut at a thickness of $10 \mu \mathrm{m}$ and mounted on a PEN membrane slide (Leica, Wetzlar, Germany). The cryosections were dehydrated with $75 \%$ ethanol for $30 \mathrm{~s}$, followed by $95 \%$ ethanol for $30 \mathrm{~s}, 100 \%$ ethanol for $30 \mathrm{~s}$, and xylen for $2 \mathrm{~min}$. After air-dried laser-capture microdissection (LCM) was performed using a Leica LMD 6000 microscope to collect 20 dye-labeled cells or non-labeled cells, separately, into Eppendorf tubes containing $\beta$-mercaptoethanol and buffer RLT (Supplementary Fig. S1). After collecting these cells, the sample tubes were immediately vortexed. Total RNA was isolated from the sample with an RNeasy Plus Micro Kit (QIAGEN) according to the manufacturer's protocol. Then, RTPCR was performed as described above, except for the number of PCR amplification cycles ( 55 cycles).

\section{Statistical analysis}

All results are expressed as mean \pm standard errors. Comparisons among groups were made by one-way analysis of variance, followed by a post hoc Tukey test. The criterion for statistical significance was $p<0.05$.

\section{RESULTS}

\section{Bladder uptake}

The fluorescent signal of TYE 563 was confirmed in the urothelial layer 1 day after intravesical application of liposome-NGF antisense complex with a TYE 563 tag (Supplementary Fig. S2), which is in accordance with a previous report, ${ }^{11}$ indicating that antisense OND was successfully delivered into the urothelium.

\section{Cystometry}

The cystometric analysis indicated that ICI was significantly $(p=0.001)$ reduced in the cystitisliposome group $(n=6)$ compared to the shamliposome group ( $n=8$; ICI: $442 \pm 64$ and $889 \pm 73 \mathrm{~s}$, 
respectively). On the other hand, ICI was significantly $(p=0.007)$ increased in the cystitis-OND group $(n=8)$ compared to the cystitis-liposome group (ICI: $711 \pm 46$ and $442 \pm 64 \mathrm{~s}$, respectively). In comparison to the sham-liposome group, ICI did not significantly differ in the sham-OND group ( $n=7 ; p=0.56$; Fig. 1$)$. Also, other cystometric parameters were not significantly different among the four groups.

\section{Bladder weight}

Compared to the sham-liposome group $(n=8)$, the bladder weight (121 \pm 9 and $204 \pm 7 \mathrm{mg}$, respectively; $p<0.001)$ of cystitis-liposome rats $(n=6)$ was significantly heavier. On the other hand, the bladder weight of the cystitis-OND group $(n=8)$ was significantly lower than the cystitis-liposome group (168 \pm 10 and $204 \pm 7 \mathrm{mg}$, respectively; $p=0.02$ ). There was no significant difference between the sham-liposome and sham-OND groups $(n=7 ; 121 \pm 9$ and $100 \pm 2 \mathrm{mg}$, respectively; $p=0.07$; Supplementary Fig. S3).

\section{Nociceptive behavior}

The number of licking events did not significantly differ among the four groups. However, the cystitis-liposome group $(n=8)$ showed a significantly ( $p=0.002$ ) higher number of freezing events than the sham-liposome group did $(n=7 ; 18 \pm 2$ and $6 \pm 1$ events for $15 \mathrm{~min}$ after RTx treatment, respectively). In comparison to the cystitis-liposome
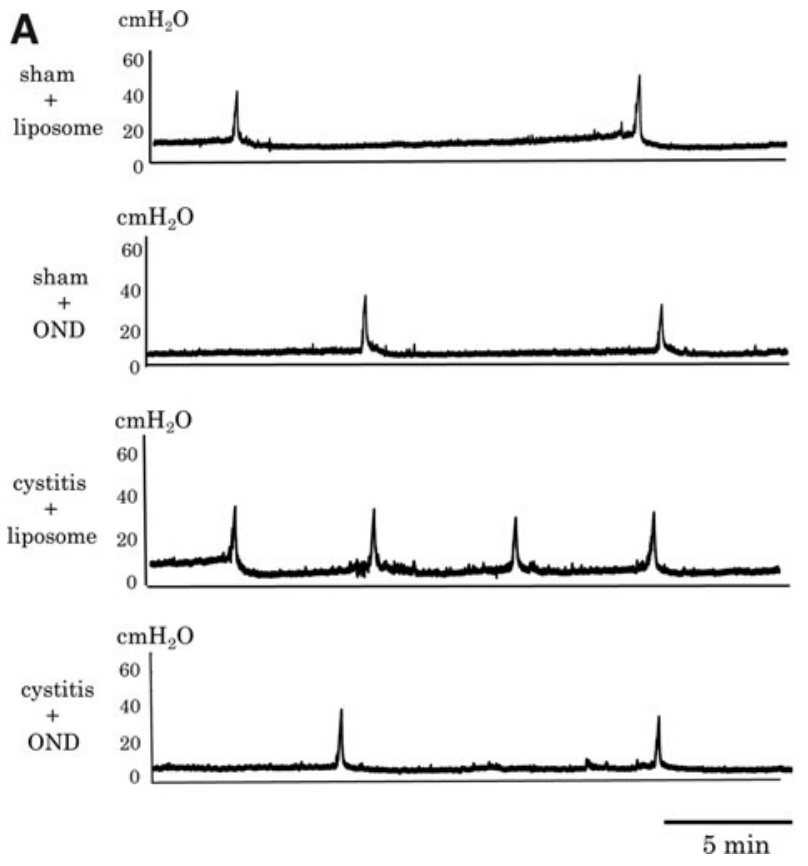

group, the number of freezing events was significantly ( $p=0.04)$ smaller in the cystitis-OND group ( $n=7 ; 18 \pm 2$ and $9 \pm 3$ events, respectively). The number of freezing events did not significantly differ between the sham-liposome and sham-OND groups ( $n=8$; Fig. 2 ).

\section{Hematoxylin and eosin staining}

In hematoxylin and eosin staining, substantial infiltration of inflammatory cells and submucosal bleeding in the bladder wall were seen in the cystitis-liposome group, which were alleviated in the cystitis-OND group. These histologic changes were not seen in sham-liposome or sham-OND groups (Supplementary Fig. S4).

\section{NGF staining}

In NGF immunofluorescence staining, the cystitis-liposome group $(n=5)$ showed significantly higher NGF expression in the urothelium than the sham-liposome group did $(n=5 ; 46 \pm 4 \%$ and $8 \pm 1 \%$, respectively; $p=0.02$ ). In contrast, the cystitisOND group $(n=5)$ showed significantly lower NGF expression in the urothelium than the cystitisliposome group did (18 $\pm 2 \%$ and $46 \pm 4 \%$, respectively; $p=0.002)$. Compared to the sham-liposome group, NGF expression did not significantly differ in the sham-OND group $(n=5 ; 8 \pm 1 \%$ and $5 \pm 1 \%$, respectively; $p=0.88$ ). On the other hand, the NGF expression in the detrusor was not significantly different among the four groups (Fig. 3).

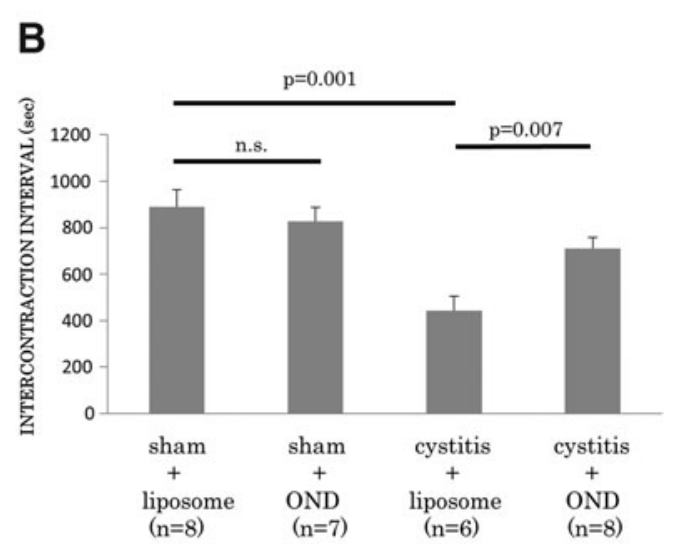

Figure 1. Results of cystometric analyses in an awake condition. Representative traces of cystometry (A) and the comparison of intercontraction intervals (B). 

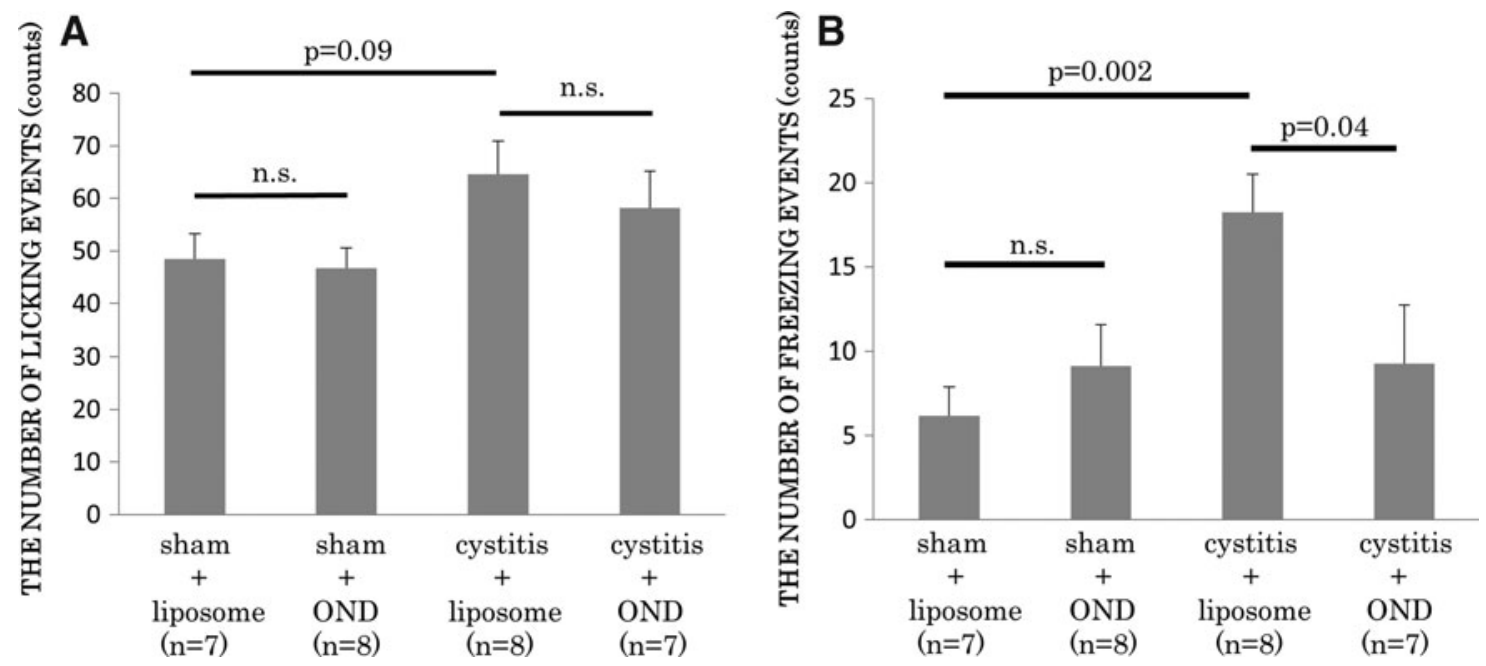

Figure 2. Resiniferatoxin (RTx)-induced nociceptive behavior. $\mathrm{RTx}(3 \mu \mathrm{M})$ was administered into the bladder through a temporary indwelled urethral catheter and kept in the bladder for $1 \mathrm{~min}$. The number of licking events (A) and freezing events (B) was counted for a 15 min period with $5 \mathrm{~s}$ intervals.

I
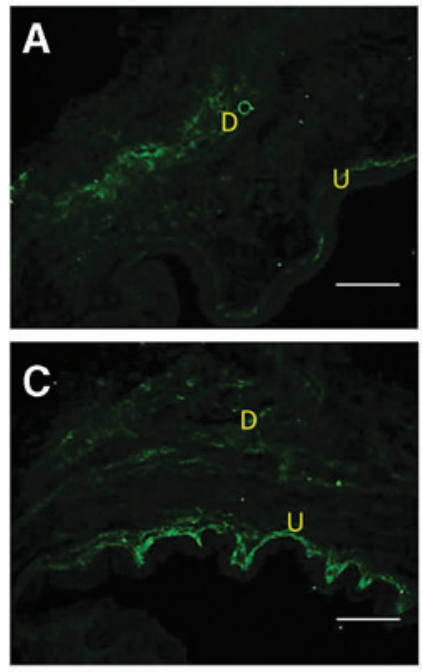
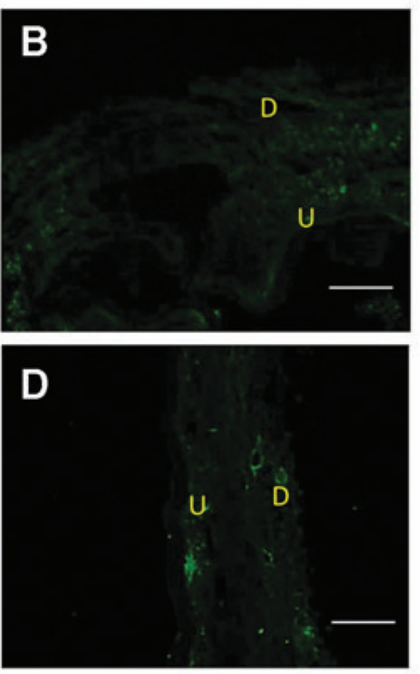

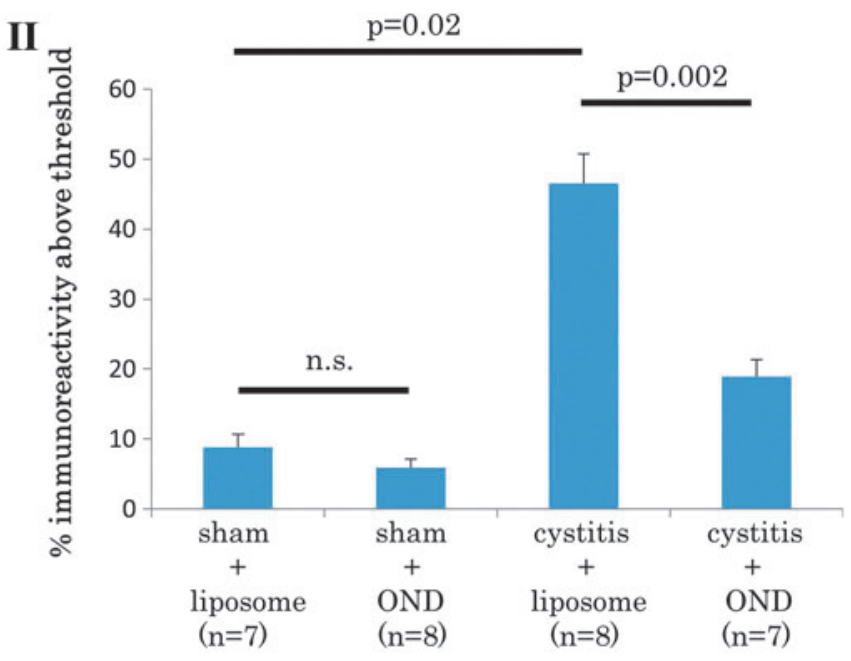

III

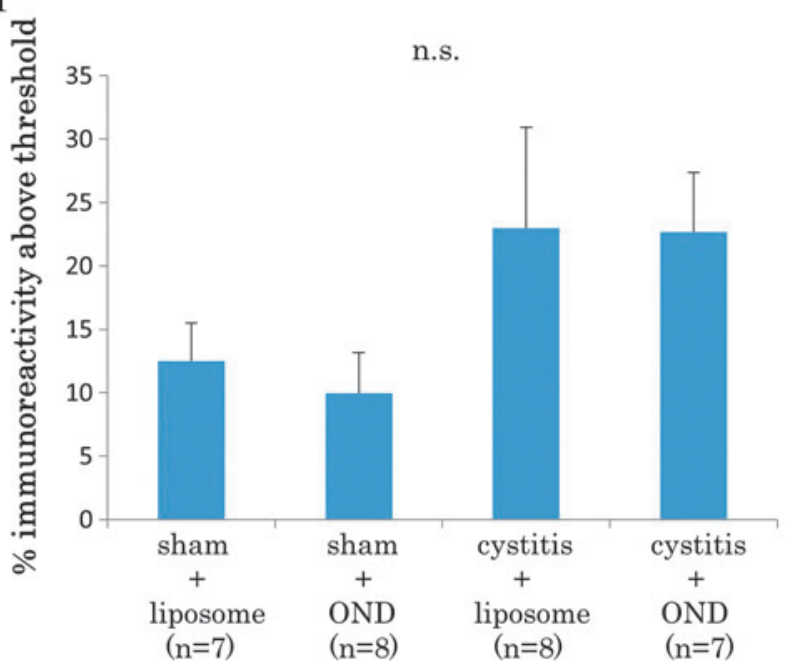

Figure 3. Nerve growth factor (NGF) immunofluorescence in the bladder. Photomicrographs of bladder sections (I) from a sham-liposome rat (A), a shamoligonucleotide (OND) rat (B), a cystitis-liposome rat (C), and a cystitis-OND rat (D). The percentage area of positive immunoreactivity above the threshold in the urothelium (II) and the detrusor (III). D, detrusor; U, urothelium. Scale bars: $200 \mu \mathrm{m}$. 
In NGF expression of L6 DRG neurons, the cystitis-liposome group showed a significantly larger number of NGF-positive cells compared to the sham-liposome group $(6.5 \pm 1.3$ and $0 \pm 0$ cells/section, respectively; $p=0.01$ ). However, the cystitisOND group showed a significantly smaller number of NGF positive cells in comparison to the cystitisliposome group $(0.5 \pm 0.4$ and $6.5 \pm 1.3$ cells/section, respectively; $p=0.01$ ). There was no significant difference between the sham-liposome and shamOND groups $(0 \pm 0$ and $0.5 \pm 0.4$ cells/section, respectively; $p=0.35$; Fig. 4 ).

In addition, it was confirmed that the positive staining above the threshold level was not observed in the urothelial or muscle layer of the bladder or DRG neurons when tissue sections were stained without NGF antibodies (data not shown), although autofluorescence due to the edge effect was sometimes seen, especially in DRG sections (Fig. 4)

\section{Gene expression in the bladder}

RT-PCR showed that the expression of $N G F$ and TRPV1 mRNA in the mucosa was significantly higher in the cystitis-liposome group than it was in the sham-liposome group ( $p=0.001$ and 0.03 , respectively). On the other hand, these gene expressions in the mucosa were significantly lower in the cystitis-OND group than they were in the cystitisliposome group ( $p=0.007$ and 0.02 , respectively). The expression of $N G F$ and TRPV1 mRNA in the mucosa did not significantly differ between the sham-liposome and sham-OND groups. In the detrusor, the cystitis-liposome group showed significantly ( $p=0.03$ ) higher expression of NGF mRNA than the sham-liposome group did, whereas the cystitis-OND group did not show a significant difference compared to the cystitis-liposome group ( $p=0.5$ ). The expression of TRPV1 mRNA in the detrusor did not significantly differ among the four groups (Fig. 5).

\section{Gene expression in L6 DRG}

In Fast Blue-labeled cells, the cystitis-liposome group showed significantly higher expression of TRPA1, P2X3, and BDNF mRNA than the shamliposome group did ( $p=0.03,0.01$, and 0.001 , respectively). In contrast, the cystitis-OND group showed significantly lower expression of mRNA of these molecules than the cystitis-liposome group $\operatorname{did}(p=0.04,0.006$, and 0.03 , respectively). There were no significant differences in expression between the sham-liposome and sham-OND groups. In addition, there was no significant difference in TRPV1 mRNA expression among the four groups (Fig. 6). In non-labeled DRG cells, there were no significant differences in any of these molecules' mRNA expression among the four groups (data not shown).

\section{DISCUSSION}

The results of this study indicate that a single intravesical injection of HP increased: (i) the number
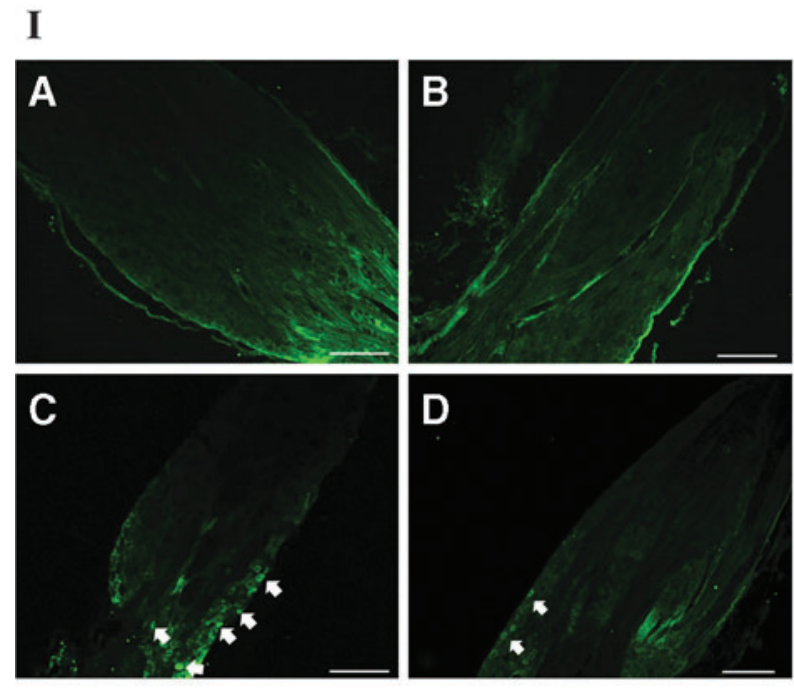

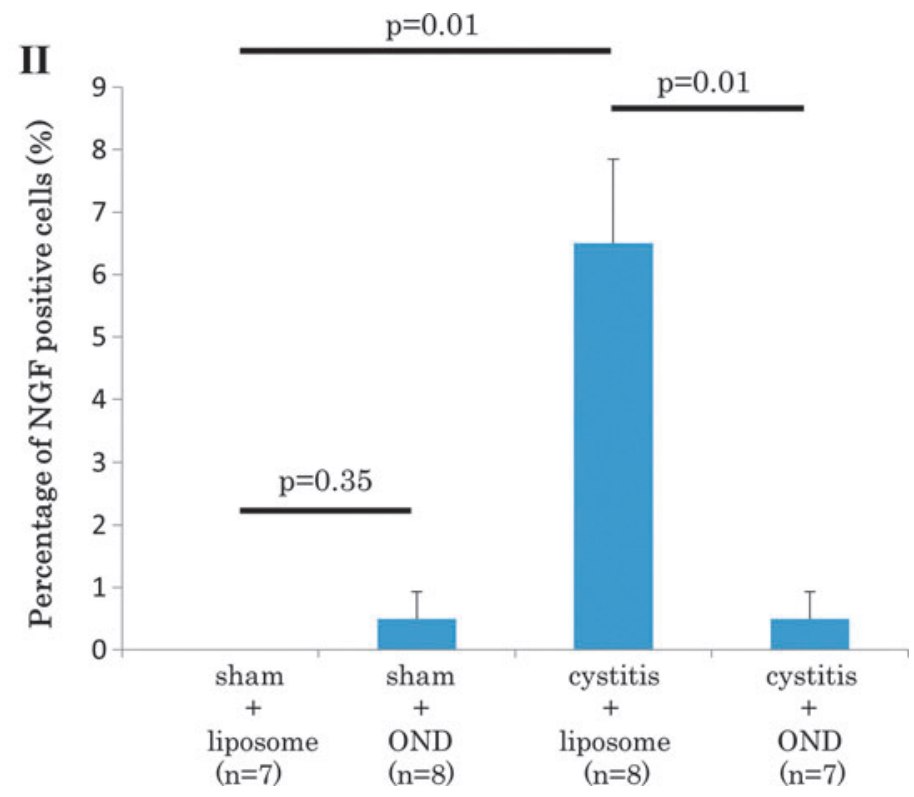

Figure 4. NGF immunofluorescence in L6 DRG. Photomicrographs of L6 DRG sections (I) from a sham-liposome rat (A), a sham-OND rat (B), a cystitisliposome rat (C), and a cystitis-OND rat (D). The percentage ratio of the number of NGF-positive cells relative to the total cell count in L6 DRG sections (II). Arrows indicate NGF-positive neurons. Scale bars: $100 \mu \mathrm{m}$. 
A NGF in the Mucosa

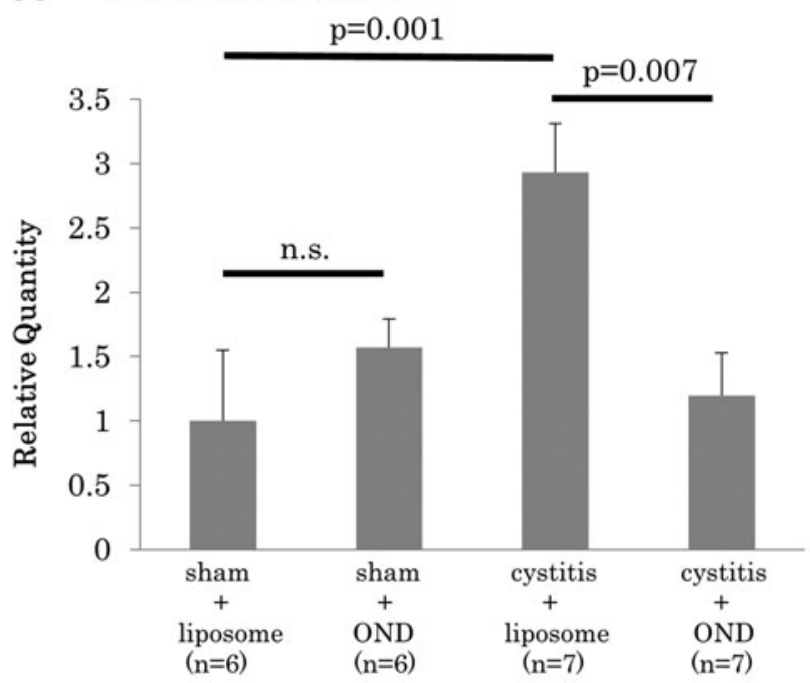

C NGF in the Detrusor

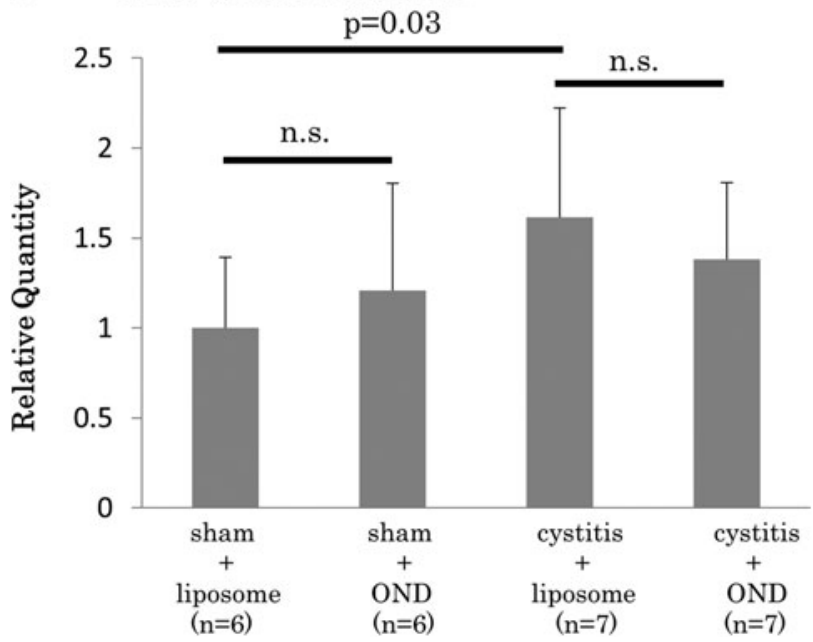

B TRPV1 in the Mucosa

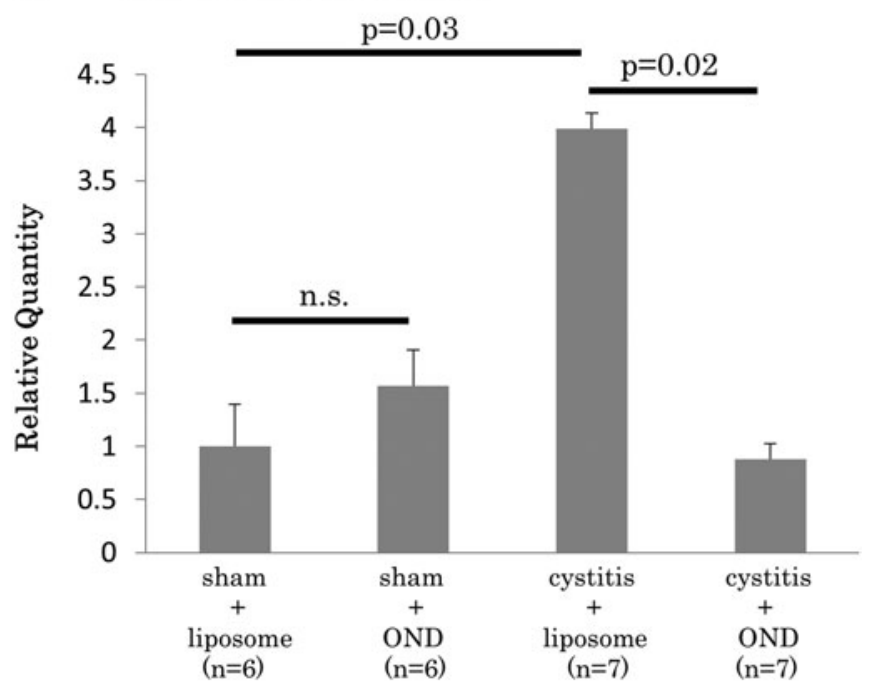

D TRPV1 in the Detrusor

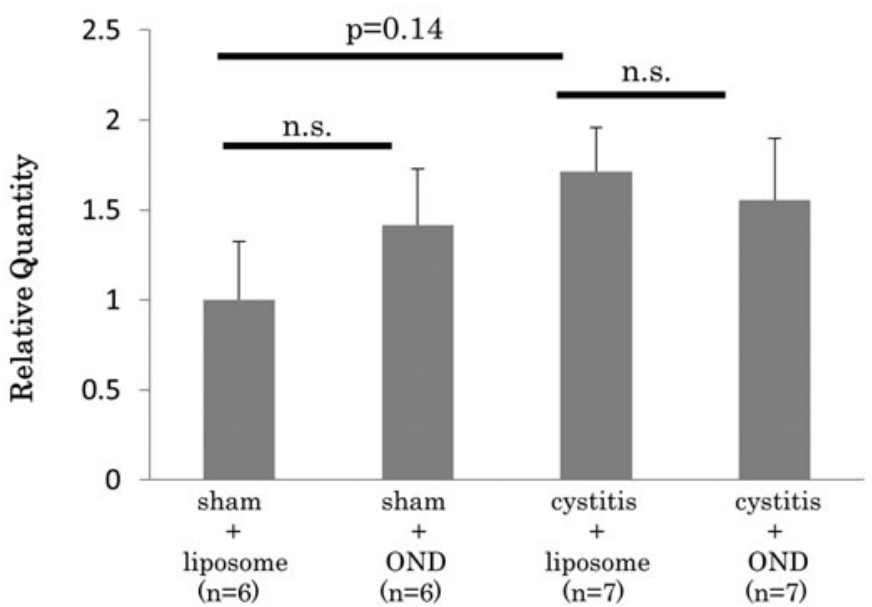

Figure 5. Expression levels of NGF mRNA (A) and TRPV1 mRNA (B) in the bladder mucosa, and NGF mRNA (C) and TRPV1 mRNA (D) in the detrusor.

of micturition during cystometry, (ii) the number of RTx-induced freezing events, (iii) bladder weight as well as infiltration of inflammatory cells and submucosal bleeding in the bladder, (iv) increased NGF protein expression in the mucosa and L6 DRG, and (v) increased $N G F$ and TRPV1 mRNA expressions in the bladder mucosa, as well as TRPA1, $P 2 X 3$, and $B D N F$ mRNA expression in bladder afferent neurons from L6 DRG. Moreover, it was found that intravesical administration of liposomes complexed with antiNGF antisense OND improved all of the HP-induced pathophysiological findings described above.

$\mathrm{HP}$ is a known reactive oxygen species, which leads to lipid peroxidation and oxidation of DNA and proteins. ${ }^{23}$ Using mice, Homan et al. reported that a single intravesical injection of HP damaged the bladder urothelium to increase permeability of the urothelial barrier and inflammation in the bladder. ${ }^{15}$ Therefore, it is assumed that HP induced bladder inflammation via urothelial damage that was caused by lipid peroxidation and oxidation of DNA and proteins. A previous study also showed that reactive oxygen species activate inflammasomes through mitogen-activated protein kinases and extracellular signal-regulated protein kinases 1 and 2 and that inflammasome activation leads to pyroptosis, a process of programmed cell death distinct from apoptosis, and stimulation of further downstream targets such as interleukin (IL)- $1 \beta$ and IL-18, leading to activation of an inflammatory cascade. ${ }^{24}$ Hence, another possibility is that HP causes bladder inflammation via inflammasome activation in HP-induced cystitis. In addition, it has been shown that several stimuli such as TNF- $\alpha$, 
A TRPV1 in L6 DRG

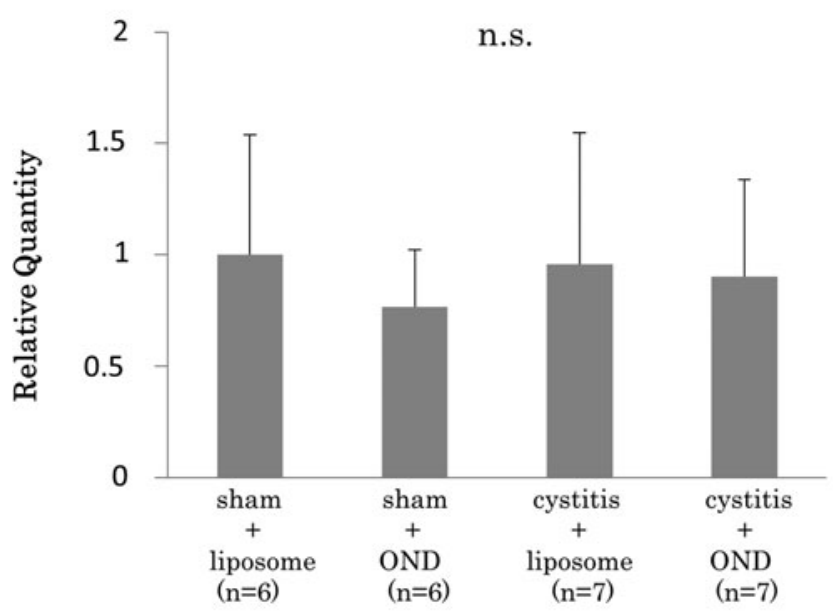

C P2X3 in L6 DRG

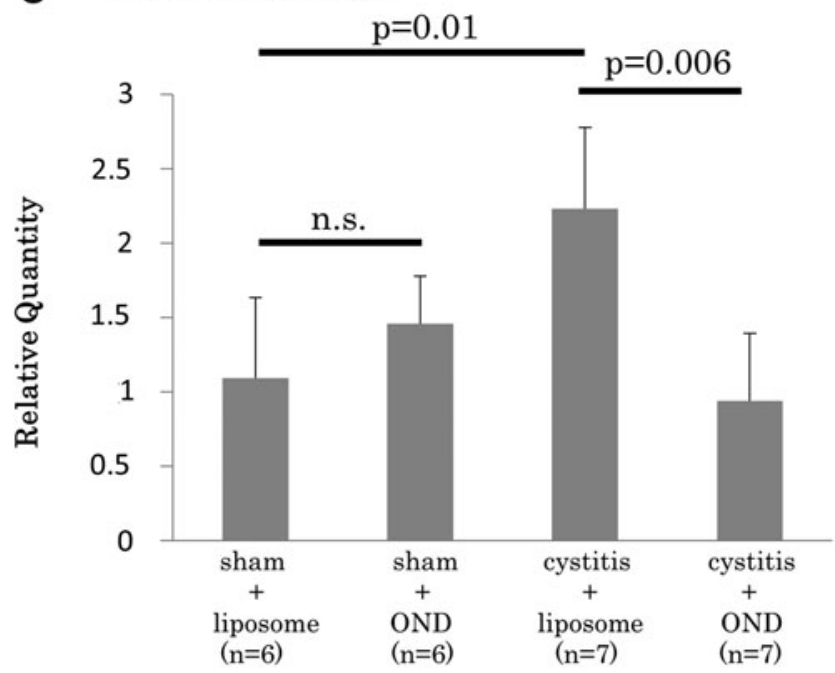

B TRPA1 in L6 DRG

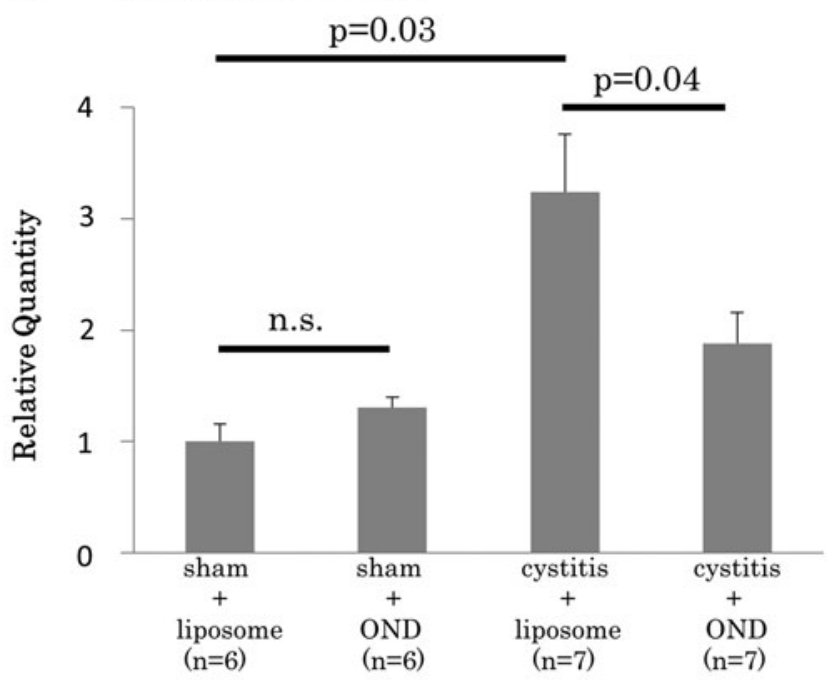

BDNF in L6 DRG

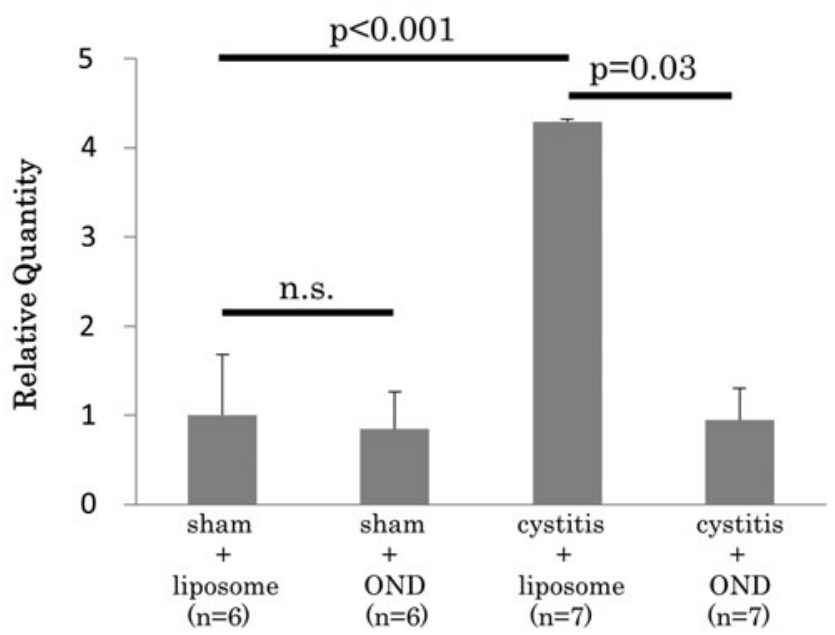

Figure 6. Expression levels of TRPV1 mRNA (A), TRPA1 mRNA (B), P2X3 mRNA (C), and BDNF mRNA (D) in bladder afferent neurons obtained from L6 DRG.

interleukin-1 $\beta$, or transforming growth factor beta regulate NGF expression in astrocytes, Schwann cells, fibroblasts, muscle cells, and neurons. ${ }^{8}$ Therefore, it is possible that bladder inflammation enhances NGF expression via activation of chemical mediators, including cytokines, although further studies are needed to clarify these points. Moreover, $\mathrm{NGF}$ is known as an activator of immune cells such as mast cells, ${ }^{25}$ lymphocytes, and monocytes/macrophages, which express NGF and TrkA receptors. ${ }^{26}$ Thus, it is likely that an increase in NGF expression further aggravates bladder inflammation in HPinduced cystitis.

There has been increasing evidence to show that NGF elicits nociceptive sensation in the bladder as well as bladder overactivity through increased excit- ability of C-fiber afferent pathways. In basic research, overexpression of NGF in the mouse urothelium reportedly leads to pelvic hypersensitivity and bladder overactivity in NGF-overexpressing transgenic mice. ${ }^{27}$ It was also found that there was a marked increase in densities of CGRP- and substance Ppositive C-fiber afferent fibers in the bladder of transgenic mice. A previous study also showed that continuous infusion of NGF into the intrathecal space at L6-S1 levels of the spinal cord decreased the density of slowly inactivating A-type $\mathrm{K}^{+}$currents in bladder afferent neurons to enhance excitability of Cfiber afferent pathways and induce bladder overactivity. ${ }^{28}$ Therefore, it is likely that HP-induced NGF overexpression increases excitability of C-fiber afferent pathways, resulting in bladder overactivity 
and enhanced bladder pain sensitivity in this study. Moreover, previous studies indicated that NGF stimulates gene expression related to nociception such as TRPV1 receptor, ${ }^{29} T R P A 1,{ }^{30}$ or $B D N F .{ }^{31}$ TRPV1 mRNA was significantly highly expressed in the bladder mucosa after HP-induced cystitis in this study. Additionally, the expression of TRPA1, P2X3, and $B D N F$ mRNA was significantly higher in bladder afferent neurons from L6 DRG of HP-induced cystitis rats. Because it is known that NGF protein is retrogradely transported from target organs to DRG ${ }^{32-34}$ these gene expressions were possibly upregulated by transported NGF proteins in L6 DRG. Therefore, it is likely that NGF-induced overexpression of these nociception-related genes in the bladder as well as in bladder afferent neurons also contribute to bladder overactivity and enhanced pain behavior after HP-induced cystitis. Taken together, rats with HP-induced cystitis exhibit several aspects similar to IC/BPS in human such as urinary frequency, bladder pain sensation, and upregulation of NGF, TRPV1, and TRPA1. ${ }^{35}$ Therefore, this model seems to be suitable to perform a proof-of-concept study that evaluates the effect of intravesical treatment of NGF antisense OND complexed with liposome that could be applicable for the symptom relief in IC/BPS patients.

In this study, the bladder uptake experiment showed that antisense OND was successfully delivered into the urothelium but not into the detrusor, as similarly found in a previous study. ${ }^{11}$ These findings coincide with the results of RT-PCR and immunohistochemistry, which showed that the antisense OND treatment significantly reduced NGF mRNA and protein overexpression induced by HP in the bladder mucosa but not in the detrusor. Furthermore, although it did not reduce $N G F$ mRNA in the detrusor, the antisense OND therapy still dramatically improved HP-induced bladder overactivity and enhanced nociceptive behavior. It has also been reported that the urothelium is a major source of NGF in the bladder. ${ }^{36-38}$ Taken together with the present results, NGF in the urothelium plays a more important role than the detrusor NGF for the development of bladder overactivity and nociception. Compared to the cystitis-liposome group, the cystitis-OND group also showed significantly lower bladder weight, as well as fewer inflammatory changes in the bladder. As mentioned above, because NGF acts as an inflammatory mediator to worsen tissue inflammation, it is likely that the antisense OND therapy reduces bladder inflammation via blockade of NGF production. Additionally, the intravesical antisense OND therapy significantly reduced expressions of TRPV1 mRNA in the mucosa as well as TRPA1, P2X3, and
BDNF mRNA in bladder afferent neurons obtained from L6 DRG, even though the inhibitory effects of NGF production were limited in the urothelium. Also, as mentioned above, NGF is retrogradely transported from target organs to DRG where it upregulates gene expression related to nociception. Therefore, the therapeutic effects of intravesical antisense OND treatment on bladder hypersensitivity in HP-induced cystitis rats could be at least in part due to the downregulating of such gene expressions in afferent neurons via inhibition of the NGF transport into DRG. In addition, in this study, the normalization of nociception-related gene expression after HP instillation was found only in bladder afferent neurons but not in non-bladder afferent neurons. The effects of liposome-based NGF antisense therapy are therefore likely to be limited in the bladder and its afferent pathways. Thus, it can be expected that the local suppression of NGF by liposome-based NGF antisense delivery into the bladder would avoid systemic adverse effects.

Finally, the present study demonstrated that there was no significant difference in any experimental parameters between the sham-liposome and sham-OND groups, including the baseline NGF expression, whereas the NGF antisense OND treatment can reduce the inducible production of NGF as well as bladder hypersensitivity after cystitis. Thus, these results suggest that the liposomebased anti-NGF gene therapy in the bladder could be beneficial for the treatment of disease conditions such as IC/BPS without affecting the normal, baseline bladder function and NGF expression.

There are some limitations to this study. First, it focused on the NGF-mediated sensory and pain mechanisms because NGF is reportedly a major mediator that induces sensitization of bladder afferent pathways underlying the lower urinary tract dysfunction and pain. ${ }^{8}$ However, NGF is also involved in the modulation of immune responses as described above, and this study showed that infiltration of inflammatory cells after HP-induced cystitis was reduced by the NGF antisense therapy. Thus, further studies are needed to clarify the effects of liposomebased anti-NGF gene therapy on immune responses induced after bladder inflammation. Second, the effects of NGF antisense therapy were evaluated on mRNA levels of nociception-related receptor genes in laser-captured bladder afferent neurons but not their protein levels, because the protein amount extracted from a limited number of laser-captured neurons was not high enough for Western blot analysis. Thus, another series of experiments such as immunohistochemistry is planned in the future to confirm that the changes in protein expression occur similarly to those 
in gene expression. Third, only the molecular expression changes in bladder afferent pathways were evaluated in this study. Thus, a functional study to investigate the electrophysiological properties of bladder afferent neurons, including slowly inactivating A-type $\mathrm{K}^{+}$channel activity, is currently ongoing.

\section{CONCLUSIONS}

The present study indicated that the site-specific reduction of NGF ameliorated bladder hypersensitivity induced by HP cystitis. Intravesical appli- cation of liposome-NGF antisense complex could be a useful treatment that can obviate systemic toxicity for hypersensitive bladder disorders such as IC/BPS.

\section{ACKNOWLEDGMENT}

Our research was funded by DOD W81XWH-121-0565.

\section{AUTHOR DISCLOSURE}

No competing financial interests exist.

\section{REFERENCES}

1. Chancellor MB, Yoshimura N. Treatment of interstitial cystitis. Urology 2004:63:85-92.

2. Moutzouris D-A, Falagas ME. Interstitial cystitis: an unsolved enigma. Clin J Am Soc Nephrol 2009;4:1844-1857.

3. Hayashi $Y$, Takimoto $\mathrm{K}$, Chancellor MB, et al. Bladder hyperactivity and increased excitability of bladder afferent neurons associated with reduced expression of Kv1.4 alpha-subunit in rats with cystitis. Am J Physiol Regul Integr Comp Physiol 2009:296:R1661-1670

4. Yoshimura N, de Groat WC. Increased excitability of afferent neurons innervating rat urinary bladder after chronic bladder inflammation. J Neurosci 1999:19:4644-4653

5. Yoshimura N, Oguchi T, Yokoyama $\mathrm{H}$, et al. Bladder afferent hyperexcitability in bladder pain syndrome/interstitial cystitis. Int J Urol 2014; 21:18-25

6. Homma Y. Hypersensitive bladder: a solution to confused terminology and ignorance concerning interstitial cystitis. Int J Urol 2014;21: 43-47.

7. Sofroniew MV, Howe CL, Mobley WC. Nerve growth factor signaling, neuroprotection, and neura repair. Annu Rev Neurosci 2001;24:1217-1281.

8. Ochodnicky P, Cruz CD, Yoshimura N, et al. Neurotrophins as regulators of urinary bladder function. Nat Rev Urol 2012;9:628-637.

9. Qu HC, Zhang $W$, Yan $S$, et al. Urinary nerve growth factor could be a biomarker for interstitial cystitis/painful bladder syndrome: a meta-analysis. PLoS One 2014;9:e106321

10. Fujita M, Kasai E, Omachi S, et al. A novel method for assessing bladder-related pain reveals the involvement of nerve growth factor in pain associated with cyclophosphamide-induced chronic cystitis in mice. Eur J Pain 2016;20:79-91.

11. Kashyap M, Kawamorita N, Tyagi V, et al. Downregulation of nerve growth factor expression in the bladder by antisense oligonucleotides as new treatment for overactive bladder. J Urol 2013; 190:757-764

12. Evans RJ, Moldwin RM, Cossons N, et al. Proof of concept trial of tanezumab for the treatment of symptoms associated with interstitial cystitis. J Urol 2011;185:1716-1721.

13. Wood JN. Nerve growth factor and pain. New Engl J Med 2010;363:1572-1573.

14. Kawamorita N, Yoshikawa S, Kashyap M, et al Liposome based intravesical therapy targeting nerve growth factor ameliorates bladder hypersensitivity in rats with experimental colitis. J Urol 2016;195:1920-1926.

15. Homan T, Tsuzuki T, Dogishi K, et al. Novel mouse model of chronic inflammatory and overactive bladder by a single intravesical injection of hydrogen peroxide. J Pharmacol Sci 2013:121:327337.

16. Dogishi K, Kodera M, Oyama S, et al. Long-lasting pain-related behaviors in mouse chronic cystitis model induced by a single intravesical injection of hydrogen peroxide. J Pharmacol Sci 2015;129:244246.

17. Takata T, Motoo Y, Tomosugi N. Effect of Saikokeishito, a Kampo medicine, on hydrogen peroxideinduced premature senescence of normal human dermal fibroblasts. J Integr Med 2014;12:495503.

18. Brown L, Ongusaha PP, Kim HG, et al. CDIP, a novel pro-apoptotic gene, regulates TNFalphamediated apoptosis in a p53-dependent manner. EMBO J 2007:26:3410-3422.

19. Abais JM, Xia M, Li G, et al. Contribution of endogenously produced reactive oxygen species to the activation of podocyte NLRP3 inflammasomes in hyperhomocysteinemia. Free Radic Biol Med 2014;67:211-220.

20. Kadekawa K, Yoshimura N, Majima T, et al Characterization of bladder and external urethral activity in mice with or without spinal cord injury - a comparison study with rats. Am J Phy- siol Regul Integr Comp Physiol 2016;310:R752758

21. Lecci A, Giuliani S, Lazzeri M, et al. The behavioral response induced by intravesical instillation of capsaicin rats is mediated by pudendal urethral sensory fibers. Life Sci 1994;55:429436

22. Saitoh C, Chancellor MB, de Groat WC, et al Effects of intravesical instillation of resiniferatoxin on bladder function and nociceptive behavior in freely moving, conscious rats. J Urol 2008;179: 359-364

23. Mates J. Effects of antioxidant enzymes in the molecular control of reactive oxygen species toxicology. Toxicology 2000;153:83-104.

24. Harijith A, Ebenezer DL, Natarajan V, et al. Reactive oxygen species at the crossroads of inflammasome and inflammation. Front Physiol 2014;5:352

25. Sawada J, Itakura A, Tanaka A, et al. Nerve growth factor functions as a chemoattractant for mast cells through both mitogen-activated protein kinase and phosphatidylinositol 3-kinase signaling pathways. Blood 2000;95:2052-2058.

26. Vega JA, Garcia-Suarez O, Hannestad J, et al Neurotrophins and the immune system. J Anat 2003:203:1-19

27. Schnegelsberg B, Sun TT, Cain G, et al. Overexpression of NGF in mouse urothelium leads to neuronal hyperinnervation, pelvic sensitivity, and changes in urinary bladder function. Am $\mathrm{J}$ Physiol Regul Integr Comp Physiol 2010;298: R534-547.

28. Yoshimura N, Bennett NE, Hayashi $Y$, et al. Bladder overactivity and hyperexcitability of bladder afferent neurons after intrathecal delivery of nerve growth factor in rats. J Neurosci 2006;26:10847-10855.

29. Jankowski MP, Koerber HR. Neuroscience neurotrophic factors and nociceptor sensitization. In: Kruger L, Light AR, eds. Translational Pain Re- 
search: From Mouse to Man. Boca Raton, FL: CRC Press/Taylor \& Francis, 2010:ch. 2.

30. Diogenes A, Akopian AN, Hargreaves KM. NGF up-regulates TRPA1: implications for orofacial pain. J Dent Res 2007:86:550-555.

31. Tirassa P, Triaca V, Amendola T, et al. EGF and NGF injected into the brain of old mice enhance BDNF and ChAT in proliferating subventricular zone. J Neurosci Res 2003;72:557-564.

32. Reynolds AJ, Hendry IA. A technique for 125Ilabelling of neurotrophins and the use of retrograde axonal transport as a bioassay. Brain Res Brain Res Protoc 1999;3:308-312.

33. Lalli G, Schiavo G. Analysis of retrograde transport in motor neurons reveals common endocytic carriers for tetanus toxin and neurotrophin receptor p75NTR. J Cell Biol 2002;156: 233-239.

34. Yip HK, Johnson EM Jr. Comparative dynamics of retrograde transport of nerve growth factor and horseradish peroxidase in rat lumbar dorsal root ganglia. J Neurocytol 1986;15:789798.

35. Homma $Y$, Nomiya A, Tagaya M, et al. Increased mRNA expression of genes involved in pronociceptive inflammatory reactions in bladder tissue of interstitial cystitis. J Urol 2013;190:19251931.

36. Liang CC, Tseng LH, Ko YS, et al. Expression of nerve growth factor immunoreactivity and mes- senger RNA in ischemic urinary bladder. Neurourol Urodyn 2010;29:512-516.

37. Tyagi P, Banerjee R, Basu S, et al. Intravesical antisense therapy for cystitis using TAT-peptide nucleic acid conjugates. Mol Pharm 2006;3: 398-406.

38. Guerios SD, Wang ZY, Boldon K, et al. Blockade of NGF and trk receptors inhibits increased peripheral mechanical sensitivity accompanying cystitis in rats. Am J Physiol Regul Integr Comp Physiol 2008;295:R111-122.

Received for publication August 4, 2016; accepted after revision December 19, 2016.

Published online: April 18, 2017 